\title{
Que Reste-T-II Des Héritiers Et De La Reproduction (1964-1971) Aujourd'hui? Questions, Méthodes, Concepts Et Réception D'une Sociologie De L'éducation
}

\author{
by Jean-Claude Passeron \\ Ecole des Hautes Etudes en Sciences Sociales
}

Sociological Research Online 12(6)13

<http://www. socresonline.org.uk/12/6/13.html>

doi:10.5153/sro. 1452

Received: 13 Oct 2006 Accepted: 14 Jun 2007 Published: 30 Nov 2007

\begin{abstract}
I have tried to recreate here some historiographical information taken from my teaching notes, from research documents from the 1960s and, in particular, from simple recollections of discussions with other researchers. There are also contained here recollections of readings of works and reviews from this period and also recollections of encounters with audiences (university or otherwise) during public debates. I am therefore working within the context of the questions that $\mathrm{J}$-L Robert asked in his introduction at the Study Day on "Concepts developed between 1945 and 1975", as well as within a context of approaches which are intended to be scientific in terms of their methodologies, questions, aims, sources and conclusions. But I am also working within the context of follow-up work spanning two decades on "the diffusion and appropriation of concepts" which essentially focussed on the sociology of education and culture. I am therefore responding to many points of fact asked by J-M Chapoulie in his introduction or of which he asked for my memories. However, I will not recall here my personal relationship with Bourdieu during our dozen or so years of close collaboration, in which a sociology of education was developed which we broadly shared and which remained so, despite our epistemological differences of opinion after 1972, into which I gave an insight in a recent article.
\end{abstract}

\section{L'abstrait}

J'ai essayé de restituer ici quelques informations historiographiques, à partir de mes notes d'enseignement, de documents de recherche des années 60 et, le plus souvent, de simples souvenirs de discussions avec d'autres chercheurs ; souvenirs aussi de lectures d'ouvrages et de compte-rendus de cette époque ou de confrontations avec des auditoires (universitaires ou non) dans des débats publics. Je me suis donc placé dans le cadre des questions posée par J.-L. Robert dans son introduction à la Journée d'études sur ' les concepts élaborés dans la période 1945-1975,' ainsi que des démarche qui se voulaient scientifiques avec leurs méthodes, leurs questions, leurs objets, leurs sources et conclusions '; mais aussi dans le cadre du suivi, sur deux décennies, de ' la diffusion et de l'appropriation des concepts ' qui concernaient au premier chef la sociologie de l'éducation et de la culture. Je réponds ainsi à plusieurs questions de fait que posait J.-M. Chapoulie dans son introduction ou sur lesquelles il a sollicité ma mémoire. Mais je n'entre pas dans le rappel de ce qu'ont été mes rapports personnels avec Bourdieu durant la douzaine d'années de collaboration étroite où s'est élaborée une sociologie de l'éducation qui nous était en gros commune et qui l'est restée, en dépit de notre divergence épistémologique après 1972, puisque je viens d'en donner un aperçu dans un article récent

\section{Introduction}

1.1 J'ai essayé de restituer ici quelques informations historiographiques, à partir de mes notes d'enseignement, de documents de recherche des années 60 et, le plus souvent, de simples souvenirs de discussions avec d'autres chercheurs ; souvenirs aussi de lectures d'ouvrages et de compte-rendus de cette époque ou de confrontations avec des auditoires (universitaires ou non) dans des débats publics. Je me suis donc placé dans le cadre des questions posée par J.-L. Robert dans son introduction à la Journée d'études sur "les concepts élaborés dans la période 1945-1975," ainsi que des démarches qui se voulaient scientifiques avec leurs méthodes, leurs questions, leurs objets, leurs sources et conclusions " (Robert 2002) ; mais aussi dans le cadre du suivi, sur deux décennies, de "la diffusion et de l'appropriation des concepts " qui concernaient au premier chef la sociologie de l'éducation et de la culture (ibidem). Je réponds ainsi à plusieurs questions de fait que posait J.-M. Chapoulie dans son introduction ou sur lesquelles il a sollicité ma mémoire. Mais je n'entre pas dans le rappel de ce qu'ont été mes rapports 
personnels avec Bourdieu durant la douzaine d'années de collaboration étroite où s'est élaborée une sociologie de l'éducation qui nous était en gros commune et qui l'est restée, en dépit de notre divergence épistémologique après 1972, puisque je viens d'en donner un aperçu dans un article récent (Passeron 2002).

\section{ENTRER EN SOCIOLOGIE DANS LES ANNÉES 50-60}

\section{1). Théories}

2.1 Reprenant l'histoire des travaux sociologiques et de leurs sources, J.-M. Chapoulie signale à juste titre que le re-départ de la sociologie française, à partir de 1945 " ne s'est pas effectué en prenant appui sur l'héritage durkheimien " (Chapoulie 1991), alors "laissé au silence et à l'abandon " ajouterais-je volontiers en citant Lévi-Strauss ${ }^{[1]}$. II a raison en ce qui concerne la plupart des champs d'enquêtes empiriques qui se sont alors ouverts en France, souvent grâce au CNRS nouvellement créé, sous l'impulsion d'universitaires comme Friedmann, Le Bras, Stoetzel, Gurvitch ou Aron, plus tard Touraine, Crozier etc. Mais en sociologie de l'Ecole ou de la mobilité sociale qui lui était liée, la question me semble se poser différemment, à cause de la place centrale que Durkheim avait accordée à l'éducation dans sa théorie des faits sociaux.

2.2 Au plan de l'organisation des concepts en théorie, l'origine des enquêtes empiriques réalisées en sociologie de l'éducation était durkheimienne au moins par leur méthode et leurs hypothèses; et cela non seulement dans notre équipe, au Centre de sociologie européenne qui revendiquait explicitement cette filiation, mais aussi dans les premiers groupes de chercheurs du CNRS dont nous étions contemporains Bourdieu et moi : Viviane Isambert-Jamati pour l'analyse sociologique des pédagogies ou chez un précurseur de la sociologie de l'enseignement technique comme Pierre Naville; de même, les premiers suivis de cohortes d'élèves dans le primaire et le secondaire par l'INED s'inscrivaient dans la lignée d'une statistique du croisement de variables que Durkheim avait intronisée en sociologie avec Le suicide (1897). Bourdieu comme moi, avions choisi le travail de rassemblement et de traitement de données d'enquêtes ou de recensements pour rompre avec le mode de réflexion que l'agrégation de philosophie imprimait alors presque irréversiblement dans l'esprit de ceux qui y avaient réussi. Un ou deux ans de préparation aux recettes de la dissertation y suffisent, plaisantions-nous, mais combien d'années de terrain, de lectures spécialisées, de traitements méthodiques de données empiriques pour pouvoir s'affranchir de ses schèmes rhétoriques et de ses modes de conceptualisation, tous également rétifs à l'esprit d'enquête. Cette formation, comme le passage par l'Ecole Normale Supérieure, détournait, à mon avis, de la recherche sociologique, aussi bien ceux qui y avaient échoué, après la rude ascèse de la khâgne, que ceux qui ne se remettaient pas mieux d'y avoir réussi, marqués eux aussi, et plus encore peut-être, par le ritualisme de cette préparation à l'érudition lettrée du passé. Dès 1955 me semble-t-il, les générations entrées dans la recherche en sciences sociales après la Libération ont commencé à répudier la vieille révérence envers les "humanités classiques " toujours aussi souverainement assurées de leur droit à régenter les sciences de l'homme, sans se frotter à leur pratique. La plupart des chercheurs de nos disciplines commençaient à subir en même temps l'influence de courants et d'écoles apparus dès l'avantguerre, qui avaient voulu donner une forme plus scientifique, moins littéraire et moins philosophique, à la construction d'une intelligibilité propre aux sciences sociales - mais en répudiant tout aussi fermement l'épistémologie positiviste des "naturalismes" et des évolutionnismes du XIX' siècle.

2.3 Excluant de nous affilier aux sectes philosophiques de la phénoménologie, de l'existentialisme ou de l'ontologie heideggérienne, comme aussi au marxisme de doctrine ou de militance - tous choix qui conduisaient alors à se fermer aux développements contemporains de la recherche scientifique - nous n'aspirions pas non plus à devenir historiens de la philosophie, malgré notre admiration de disciples pour Martial Guéroult ou Jules Vuillemin ; ni philosophes de la science malgré le rôle qu'ont joué alors Bachelard ou Canguilhem dans notre formation épistémologique. Ethnologie et psychanalyse aidant, la philosophie, prise en sa définition universitaire, glissait doucement de son piédestal, restaurée en matériaux de réemploi par Sartre autour des années 1945, avant que le philosophe s'immerge dans les mêlées morales et politiques du siècle, où il s'affirma comme le maître à penser le plus influent, du moins jusqu'en 1968. Après deux générations (et les ruptures de deux guerres mondiales), le rayonnement intellectuel de l'Ecole durkheimienne s'étiolait, en même temps que s'usait le pouvoir qui avait été le sien de renouveler les programmes de toutes les sciences sociales au début du $X X^{\circ}$ siècle, jusqu'à en devenir la science-reine. Mais, le programme d'objectivation des "faits sociaux " proposé dans les Règles de la méthode sociologique (1895) de même que la théorie de l'éducation, qui irrigue tous les travaux de Durkheim, restaient à disposition du lecteur français, conviant toujours les sociologues à décrire en priorité comment se transmettent, d'une génération à l'autre, les formes et représentations de la culture d'un groupe, à travers les diverses instances et les divers agents de la socialisation - familiale ou scolaire, diffuse ou organisée ; dans la longue durée des traditions pédagogiques ou religieuses comme dans l'histoire institutionnelle des systèmes scolaires. Décrites dans leurs traits contrastés en même temps que dans leurs parentés fonctionnelles, les différentes formes historiques de l'éducation posaient ensemble le problème des causes et des fonctions de la "reproduction "socio-culturelle, corrélatif de celui des conditions historiques dans lesquelles apparaissent les ruptures pédagogiques les plus révolutionnaires ou les plus lourdes d'effets durables sur l'ensemble de la société.

2.4 II est vrai qu'au fil de la conception, de la réalisation et du traitement des premières enquêtes réalisées au Centre de sociologie européenne (1961-1965), nos travaux manifestaient aussi une réaction contre le holisme d'une "normativité "indivisible, qui avait conduit Durkheim à situer ses analyses de l'éducation et de la socialisation au niveau de la société globale et, finalement, comme dans Les formes élémentaires de la vie religieuse (1912), dans le cadre d'une "conscience collective " indivise : c'est-à-dire au niveau des représentations partagées par tous ou des pédagogies qui avaient marqué successivement, mais d'une manière supposée uniforme, chaque phase de l'histoire d'un système scolaire dans une culture nationale . 
Nous en trouvions l'exemple le plus net (avec L'évolution pédagogique en France, 1899) dans la précision du suivi historique par Durkheim, des formalismes scolaires constitués et perpétués par l'institution française, sous des modalités certes différentes (" logiques " à l'âge des philosophies médiévales, puis "rhétoriques " après la Contre-réforme), mais toujours apparentées par leur soumission à des formes minutieusement standardisées, depuis les pédagogies scolastiques jusqu'à celles des collèges jésuites et des lycées voués au culte des " humanités classiques " ou à la rhétorique des cours magistraux des universités de la $1{ }^{\circ}{ }^{\circ}$ République.

2.5 Les attendus non-durkheimiens de nos hypothèses ne nous venaient pas des théoriciens américains alors les plus influents (Parsons, Merton) ou des anthropologues de l'école "culturaliste ", mais de la lecture de Marx et de Weber, que nous enseignions alors, Pierre Bourdieu comme moi à l'Université. Weber surtout, avec sa méthode de construction des "types-idéaux ", qui l'avait conduit à distinguer en même temps que quatre "types purs " $\mathrm{d}^{\text {" }}$ orientations de l'action", dont la composition en "types mixtes " permettait de décrire une diversité d'ethos et d'habitus, de "conduites de vie ", de groupements, de hiérarchies ou de "légitimités", de séquences variées ponctuant la "quotidianisation" du charisme ou de "tensions", historiquement variables, entre sphères d'activité. Mais Marx aussi qui - dans ses ouvrages historico-sociologiques de mi-carrière - avait mis au principe de toutes ses explications historiques une "lutte des classes", incessante, multiforme et mobile, associant ainsi intimement l'histoire sociale à l'histoire économique ; la lutte de classes n'était pas présentée dans ces textes d'analyse narrative comme une machine inexorablement réglée par une logique dialectique, mais comme une série enchevêtrée d"“ antagonismes sociaux "entre classes, fractions ou "ordres statutaires ", analysés à la fois à partir de la contradiction ou de l'alliance de leurs intérêts économiques et de celle de leurs orientations symboliques ou politiques.

2.6 II était alors généralement admis, en France, parmi les sociologues récemment convertis à l'enquête quantitative que Weber, très incomplètement et souvent mal traduit, était illisible et inutile. Quant à Marx, resté emprisonné dans la langue de bois des organisations qui s'en réclamaient ou dans les polémiques obscures des marxologues sur le "jeune Marx "et la "coupure épistémologique " des Thèses sur Feuerbach, radicale pour les uns, niée par les autres, son patronage n'inspirait jamais à ses disciples, au début des années 60 , des projets d'enquête empirique sur les antagonismes de classes, sauf sous la forme du vœu pieux. Les chercheurs marxistes se devaient de ne mentionner que par des allusions prudentes et avec les réserves d'usage les recherches contemporaines de chercheurs hétérodoxes, même lorsqu'elles pouvaient enrichir la théorie marxiste d'apports scientifiques sur le travail industriel ou la sociologie de la classe ouvrière. De là qu'à la parution des Héritiers, on ait pu voir des intellectuels marxistes, parfois sociologues, découvrir avec étonnement que cet ouvrage fondé sur des enquêtes statistiques méritait un satisfecit de principe: "Malgré ses faiblesses et ses carences théoriques ", ai-je pu lire dans un compte-rendu, notre équipe de recherche avait au moins joué le rôle utile d'un "conservatoire théorique du concept de classe sociale " abandonné par tous les autres sociologues inféodés à l'idéologie bourgeoise (sauf Gurvitch peut-être, Friedmann et Naville - mais il était marqué comme "trotskiste "ou Dumazedier, suspecté, lui, de régression populiste). Mais, dans la débauche des revivals marxistes ou marxistes-léninistes d'après 68, cette reconnaissance s'effaça vite au profit de la dénonciation mécanique du "réformisme " pédagogique de Passeron et du "carriérisme " académique de Bourdieu.

2.7 Les autres programmes théoriques qui organisaient nos enquêtes étaient des plus variés provenant de disciplines voisines, où avaient, émergé dès l'avant guerre des hypothèses ou des synthèses nouvelles. Par exemple dans l'histoire quantitative issue de Simiand et Labrousse, avec le traitement systématique des "séries "; ou dans l'histoire sociale développée par l'Ecole des Annales, avec son ambition de reconstruire une " histoire totale " en étendant ses reconstitutions de "mentalités " par l'analyse approfondie de "cas" singuliers, datés et localisés. En linguistique ou en anthropologie aussi, avec le souci, commun à ces deux disciplines en plein renouveau, d'identifier comparativement les structures synchroniques ou diachroniques des langues et des cultures. Enfin dans l'analyse sémiologique, avec son exploration des différents systèmes de "signes " ou de "symboles" dont la transparence descriptive répandit un temps dans toutes les sciences sociales le méta-langage de la "communication " ou de "l'énonciation". Ou enfin dans l'analyse économique, avec ses constructions de "modèles" mathématisés qui instituaient un tout autre rapport, entre la conceptualisation et l'observation de données empiriques, que celui de l'expérimentation en sa forme canonique.

\section{2). Enquêtes}

2.8 Au plan des méthodes de la recherche empirique, il s'agissait bien de réévaluer les effets sociologiques de l'arrivée en Europe dès 1945 des techniques et buts de l'enquête, tels que les définissait la notion américaine de survey, au sens où l'influence de Lazarsfeld la vulgarisa en France. Entre 1945 et 1960 cette sociologie qui se définissait par la standardisation des instruments de mesure avait multiplié les terrains de l'enquête sociographique, au risque parfois d'accumuler des monographies autosuffisantes. II fallait, à nos yeux, utiliser, adapter, développer les potentialités de preuve statistique qu'ouvraient les formes du questionnaire et du questionnement sur échantillons ou les recensements secondaires de sources ainsi que les technologies logico-mathématiques du traitement des variables. Mais il fallait aussi, comme l'avait fait Simiand raffinant la méthodologie durkheimienne du Suicide, associer, dans une démarche interprétative cohérente, la mesure de la "significativité statistique" des corrélations observées à la reconstitution de leur "signification socio-historique " dans le contexte des faits où on les observait. Les premières formulations de l'impossibilité (historique ou géographique) de faire varier

systématiquement, dans une analyse croisée, toutes les valeurs des variables mesurées se rencontrent, en effet, chez Simiand, qui ramassait l'irréalisme sociologique des formalismes opératoires poussés à l'absurde en prenant l'exemple d'une recherche sur les comportements de "chameaux transférés au Pôle Nord " qui les comparerait à ceux de "rennes déplacés au Sahara". Le chercheur court alors après un leurre, celui de croire pouvoir arracher à tout "contexte "l'interaction statistique de "variables pures ", en 
les dégageant de l'" effet de structure " (comme on l'a baptisé à l'INSEE) qui découle, dans le recensement d'une population réelle ou sur un échantillon non "raisonné ", de la composition "singulière " des valeurs des variables observées. Maurice Halbwachs a repris le " paradoxe de Simiand " pour dénoncer, lui aussi, l'abstraction qu'engendre la quête de la pureté statistique "comme si, pour étudier les caractères démographiques d'un pays, il fallait partir d'une population qui n'est celle d'aucun pays (...) De même que l'homo economicus, un tel homo demographicus est une abstraction trop soigneusement détachée de la réalité pour nous apprendre quoi que ce soit sur le réel " (Halbwachs 1935: 338): Autrement dit, la "statistique analytique " ne peut se substituer entièrement à la "statistique descriptive " comme instrument de connaissance des réalités sociales. L'obtention d'effets statistiquement "purs ", qui oblige à "redresser" les valeurs observées sur un échantillon ou une population, conduit, lorsqu'on en fait l'objectif unique de l'analyse, à une décomposition des interactions entre variables qui, au bout du processus de purification, devient un non-sens de statistique descriptive ${ }^{[2]}$.

2.9 D'autres influences d'auteurs plus récents transformèrent, à mesure que nous lisions leurs apports notre méthode de description et notre style d'interprétation : d'abord Richard Hoggart et Basil Bernstein pendant que nous travaillions sur les matériaux des Héritiers, puis à l'époque de La reproduction, Erwin Goffman, Anselm Strauss et d'autres sociologues "interactionnistes "; parmi les linguistes, Emile Benveniste ou Noam Chomsky, les théoriciens des "actes de langage " auxquels se référa bientôt Bourdieu dans sa tentative de fusionner le structuralisme et "l'interactionnisme symbolique ", des sociolinguistes aussi comme William Labov ou des sémiologues comme Luis Prieto avec qui je collaborai longtemps, dans nos discussions à Vincennes où je l'avais appelé, puis dans sa chaire de Linguistique générale à Genève où il m'invita souvent. Tout cela avec des préférences évidemment différentes chez Bourdieu et chez moi : l'anthropologie de Lévi-Strauss joua sans doute chez lui le rôle que joua pour moi le comparatisme généalogique de Dumézil ; en philosophie, dont nous nous défiions pourtant, Bourdieu était un familier de Leibniz, tandis que ma nostalgie de la métaphysique classique des cartésiens allait au système conceptuel et aux démonstrations déductives de Spinoza.

\section{I/ L'ETAT DE LA “ QUESTION SCOLAIRE ” EN FRANCE DANS LE DEBAT POLITIQUE ET IDEOLOGIQUE DES ANNÉES 50}

3.1 Des ouvrages comme Les héritiers (1964) et La reproduction (1971), de même qu'une constellation d'articles ou livres fortement liés à ces hypothèses, ont été élaborés au sein de l'équipe d'abord restreinte, mais rapidement élargie, qui travailla sur l'enseignement, les enseignants et les enseignés de divers âges, ordres ou établissements, à partir de 1961, dans le Centre de sociologie européenne qu'avait fondé Raymond Aron à la VI ${ }^{\circ}$ section de l'Ecole Pratique des Hautes Etudes (EPHE), devenue en 1970 Ecole des Hautes Etudes en Sciences sociales (EHESS). Bourdieu comme moi-même entrions dans ces enquêtes avec pour seule connaissance de l'état de la question scolaire le souvenir des événements ou des polémiques que nous avions observés dans les années 50 . Notre préoccupation première, sans doute mûrie ou aiguisée dans nos expériences scolaires antérieures, était bien de traiter comme un objet sociologique ce qui se présentait dans le débat public comme un nœud inextricable de problèmes de politique pédagogique, questionné depuis longtemps déjà par les responsables politiques, administratifs ou syndicaux mais aussi par quelques écrivains et essayistes et, plus récemment, par tous les spécialistes ou acteurs du système scolaire dans une polémique qui allait s'amplifiant et s'exaspérant : le débat qui, pour les technocrates ou les politiques au pouvoir, était d'abord celui du "faible rendement de l'Ecole" dans la production des qualifications professionnelles était aussi, et depuis plus longtemps, pour les enseignants et les syndicats ou partis de gauche celui de l'inégalité sociale de réussite scolaire dans les sociétés européennes, lesquelles s'étaient pourtant presque toutes donné, depuis près d'un siècle, les conditions formelles (institutionnelles et légales) de la démocratisation du recrutement de l'Ecole sans s'en donner ni les moyens réels ni une politique d'ensemble, comme cela avait été le cas au début de la III République.

\section{1/.Lectures et influences}

\section{(a) Souvenirs d'enfance.}

3.2 Des débats sur la démocratisation de l'Ecole, avant 1939, Bourdieu comme moi n'avions au départ qu'une connaissance parcellaire, parfois puisée chez des historiens, mais portant alors sur les aspects les plus généraux de l'histoire des idées au début du XX siècle. Elle provenait surtout du souvenir des expériences de notre enfance d'écoliers, de lycéens, d'étudiants ou de jeunes enseignants. Par exemple, le souvenir de la polémique pédagogique portant sur la valeur formatrice des filières "classiques " ou "modernes" dans les lycées, ou le débat sur le "tronc commun " au collège, qui s'étaient enflammés, dès l'avant-guerre, du fait de leurs implications politiques ou culturelles et dont nous avions recueilli les échos durant nos études dans l'entourage familial et dans quelques commentaires entendus à la radio ou lus dans la presse des syndicats d'enseignants du primaire ou des programmes de gauche (Plan LangevinWallon à la Libération). Dès l'avant-guerre, était en effet connues, discutées - mais plus rarement chiffrées - l'inégalité de réussite scolaire des élèves de différentes origines sociales et leur orientation quasi-obligatoire vers une filière ou l'autre, lors du passage d'un cycle au suivant. Mais par quelle force (familiale, pédagogique, pécuniaire, culturelle - génétique même, soutenaient certains - , sur quels critères d'évaluation, sur quels mécanismes ce partage et cette reproduction, entre élites dominantes et masses écartées de l'enseignement supérieur s'opéraient-il ? Evoluaient-ils historiquement dans le sens d'un processus de "démocratisation "? La distribution sociale des chances scolaires se reproduisait-elle à l'identique, une fois mesurés et circonscrits quelques phénomènes marginaux de mobilité ascendante pour les uns ou de déclassement pour les autres? Et, dans les deux cas, sur quels facteurs explicatifs le partage entre reproduction et mobilité reposait-il ?

3.3 Bourdieu et moi avions eu, en gros, la même trajectoire de mobilité sociale, ayant suivi parallèlement 
les mêmes cursus scolaires, ayant entendu au lycée comme à l'université les mêmes discours de nos maîtres sur les vertus démocratiques de la scolarisation face aux vices d'une société bloquée sur ses hiérarchies: "Ecole républicaine du mérite et du talent " ou "Ecole émancipatrice du peuple ", égalité scolaire des enfants dès leur entrée à l'école et neutralité sociale de l'enseignement, dès lors qu'il était devenu laïque, obligatoire et gratuit, "vocation " ou " mission " sociale des enseignants. Bourdieu s'insurgeait contre la " bonne conscience " morale et la conviction, imperméable aux faits et aux chiffres, avec laquelle les enseignants adhéraient aux argumentations inscrites dans l'idéologie professorale de leur corps professionnel, que ce soit en sa version ethnocentriquement élitiste ou en sa version utopiquement révolutionnaire. J'étais personnellement plus attentif au rôle explicatif que jouait le consentement des familles à l'autorité de l'école dans les classes populaires ou petite-bourgeoises comme la prédisposition des parents ouvriers ou paysans à reconnaître, fût-ce à leur détriment, la neutralité ou la légitimité des sanctions de l'Ecole que ces classes opposaient à l'injustice de l'ordre social fonctionnant en son amont et son aval. La proximité théorique de notre questionnement sociologique sur l'Ecole s'alimentait ainsi à deux humeurs politiques différentes. La trajectoire antérieure d'un individu ne constitue pas, en effet, le tout de son humeur intellectuelle à l'entrée dans la vie, pas plus chez les étudiants que chez les autres adolescents, et pas plus chez les apprentis-chercheurs que chez les apprentis-bourgeois. Issu du même cursus scolaire que Bourdieu je croyais pourtant un peu plus que lui aux vertus à la fois démocratiques et intellectuelles de la formation scolaire ; j'attendais avant tout de l'enquête sociologique sur les modalités de la sélection scolaire qu'elle éclaircisse les voies d'une réforme de l'Ecole qui rapprocherait sa réalité sociologique de son idéal proclamé ; non qu'elle serve à y trouver le bouc émissaire de tous les maux de la société. Dans Les héritiers comme dans La reproduction, ces deux analyses critiques se trouvaient associées par notre effort commun d'écrire ensemble, dans le même langage théorique, le bilan de nos enquêtes sociologiques.

3.4 Personnellement, je me souviens avoir observé quotidiennement à l'extérieur du lycée - au sein duquel je voyais se snober les élèves et maîtres des sections classiques et modernes - en regardant de l'autre côté d'un mur bornant la cour de mon lycée de la cour plus turbulente d'un établissement "d'enseignement primaire supérieur" (EPS) qui conduisait au butoir du Brevet. Je commençais à être frappé par les différences de socialisation et d'apprentissage entre ces deux filières de décantation des avenirs sociaux, au sein des groupes de pairs qu'elles encadraient et modelaient. Liée professionnellement et idéologiquement à l'école primaire dont les instituteurs tentaient de garder "les meilleurs "élèves dans leur mouvance culturelle, cette deuxième école - qui ne sera absorbée qu'artificiellement dans celle des lycées et collèges par des réformes institutionnelles, plus administratives que pédagogiques, qui s'opéreront dans les années 60 - l'enseignement primaire supérieur faisait valoir par un autre personnel enseignant, à destination d'autres couches sociales, sa vocation d'ascenseur social à petite vitesse, qui n'impliquait pas, aux yeux des instituteurs, pour la plupart associés aux combats politiques des classes populaires, le risque de trahir sa classe d'origine en accédant par le Baccalauréat, à la bourgeoisie des professions libérales, des hauts fonctionnaires ou des intellectuels arrivés. Ainsi s'organisait, par l'étroite liaison entre les valeurs d'une Ecole, dont la hiérarchie se démultipliait à l'infini, et les aspirations des parents appartenant à différents groupes sociaux, la préparation de deux adolescences à des destins différents et profondément intériorisés jusqu'à être ressentis comme "naturels ". Pour l'adolescence des " héritiers " et de quelques " boursiers ", c'était une préparation toute naturelle à l'entrée dans la bourgeoisie, petite, moyenne ou grande; et pour l'autre adolescence, une résignation tout aussi naturelle, sinon à reprendre le travail ouvrier, rural ou artisanal de leurs parents, du moins à s'identifier au projet réaliste d'accéder rapidement aux professions subalternes ou intermédiaires. Ce fut ma première expérience d'une "injustice sociale", pour moi incompréhensible alors, puisque je reconnaissais, comme condisciples de la même école primaire ou anciens copains de jeux dans la cour voisine, la plupart de ceux à qui ni familles ni enseignants n'avaient proposé de tenter l'examen d'entrée en 6 ème, alors que beaucoup me semblaient le " mériter " intellectuellement autant que moi ; je ne voyais d'autre explication à mon privilège de trajet scolaire qu'un hasard de transmission culturelle : le goût de la lecture associé au désir de réussir à l'école que m'avait transmis, sans que je les choisisse, ma mère institutrice.

(b) Echos

3.5 Nous avions entendu, dans la conversation de spécialistes ou de militants de l'éducation, l'écho de travaux réalisés par des historiens ou des sociologues, par exemple à propos de la " mobilité sociale en deux générations " qui définissait entre les deux guerres une forme de mobilité sociale préférentielle pour les enfants du peuple : grands-parents appartenant aux classes populaires urbanisées ou à la paysannerie par les deux lignées ; parents petits fonctionnaires ou instituteurs ; enfants accédant à l'enseignement supérieur et par là à des professions quasi-bourgeoises - c'était précisément le cas de Bourdieu comme de moi-même. Nous avions enfin beaucoup entendu parler, jusqu'à être agacés par cette autosatisfaction, de la "vocation " indispensable à l'exercice du métier d'instituteur ou de professeur du secondaire, généralement par les intéressés eux-mêmes ; les grands universitaires quant à eux n'employaient guère alors le langage du dévouement moral ou des valeurs politiques de la "démocratisation "; ils se présentaient plutôt en dépositaires d'une mission quasi-religieuse au service du progrès scientifique et, dans les disciplines littéraires, en garants des valeurs de la haute culture " humaniste ". De même on entendait déjà parler des caractéristiques des "familles éducogènes " comme de la genèse de la "docilité scolaire " de certains élèves par opposition à l'insubordination caractérielle des autres, mais ces problèmes n'étaient alors posés que dans des travaux de psychologie ou de psychologie sociale, jamais de sociologie ou de psychanalyse. Ce n'est qu'après 1945 que la "sociologisation" de la plupart des champs de recherche, étendit la description à toutes les différences sociales dans les pratiques culturelles, par exemple avec la "sociologie des loisirs" que développa alors Joffre Dumazedier et qui favorisa la multiplication des associations porteuses de projets d'“ éducation populaire " comme Peuple et culture ou l' École des parents.

c) Rétrospections 
3.6 Dès que nous sommes revenus en sociologues sur le passé des questions qu'on commençait à appeler celles de la " démocratisation de l'Ecole " nous avons évidemment découvert toutes sortes de précurseurs, jusque fort tôt dans le $\mathrm{XIX}^{\circ}$ siècle français ou européen. De là que nous ayons souvent utilisé, pour imager nos conceptualisations, des ouvrages écrits à des fins de persuasion par des auteurs mixtes, à la fois connus et délaissés par les spécialistes, comme ceux de Goblot (philosophe et logicien, mais aussi essayiste, dans son brûlot de 1925, analysant le paradoxe du baccalauréat français, à la fois " barrière " sociale et " niveau " de culture supposé défini sur critères purement scolaires) ou de Thibaudet (critique littéraire ; 1927) sur la place singulière de l’École dans la vie intellectuelle française, tous à vrai dire consacrés aux effets culturels de l'Ecole plus qu'à la sociologie de son recrutement ou des mécanismes et conséquences de la réussite scolaire. Plus importante a sans doute été pour nous la lecture de Thorstein Veblen dans la genèse des Héritiers : la fonction de la "dépense ostentatoire de temps " dans la leisure class américaine était fort proche de notre analyse du goût de la gratuité dans les choix culturels et scolaires la bourgeoisie française; peut-être cette proximité même nous incita-t-elle à ne citer Veblen que parcimonieusement... Je m'étais aussi constitué une collection d'ouvrages ou d'articles des muckrackers (remueurs de fange), journalistes férus de reportages dans les bas-fonds des grandes villes américaines, sociologues de terrain que refoula vite le souci de dignité méthodologique de la survey aseptisée, caractéristique de la sociologie des grandes universités américaines : ils furent pourtant les premiers sociologues de la société industrielle aux Etats-Unis, dont est précisément issu Veblen. Plus généralement, nous nous sommes alors intéressés aux " romans de mobilité sociale " ou de mœurs, trame explicite, souvent inaperçue, au cœur des "romans de formation " du XIX siècle, et déjà des " romans picaresques" des XVII et XVIII ${ }^{\circ}$ siècles. L'opposition, devenue classique dans le vocabulaire français, entre boursiers et héritiers venait, bien sûr, du lexique de la description romanesque d'un des premiers effets de l'Ecole républicaine perçus par ses contemporains. Ajoutons-y l'autobiographie prise comme matière littéraire chez Jules Vallès et les romans de l'ascension sociale ou du déclassement, sans oublier ceux du " déracinement " (Barrès), ou encore les intrigues et les rencontres qui accompagnaient, dans les "romans sociaux" les cheminements originaux de personnages s'éloignant de leur milieu populaire ou provincial de naissance : thème qui remontait au moins au Julien Sorel de Stendhal et aux aventures balzaciennes d'un Rastignac ou d'un Rubempré, d'un vermicellier comme le Père Goriot ou d'un colonel Chabert.

3.7 Parallèlement, j'avais alors esquissé un travail sur l'autodidaxie, les romanciers prolétariens ou populistes du début du XX ${ }^{\circ}$ siècle, dont on trouvera des échos dans mon débat avec Grignon sur la distinction entre " misérabilisme ", " populisme " et "légitimisme " (Grignon \& Passeron 1989) ${ }^{[3]}$ comme dans ma lecture sociologique des remémorations autobiographiques de Hoggart (Passeron 1999). À partir de 1960, Bourdieu et moi nous trouvions aussi confrontés à une analyse strictement économique des fonctions et dysfonctionnements de l'Ecole : faible productivité d'une Université qui ne retrouvait dans ses diplômés que 60 \% de ses inscrits, chômage des diplômés dans certaines disciplines et inutilité professionnelle de leurs diplômes remettaient en cause la rentabilité des investissements publics dans l'éducation. Dans ce flot de critiques technocratiques indifférentes à la sociologie et à l'histoire de l'éducation et de la socialisation, je fus surtout frappé par un petit manifeste de Vermot-Gauchy, catéchisme simplifié de la vulgate productiviste appliquée à une Ecole supposée servir une fonction unique (Vermot-Gauchy 1965), auquel j'essayai de répondre en lui opposant une autre prospective de la réforme de l'Université [4], celle bien mieux informée sociologiquement du Rapport Robbins qui, en GrandeBretagne, distinguait au moins, pour caractériser sa prévisibilité incertaine à long terme, la " demande technique " de la " demande sociale " d'éducation, inscrite, elle, dans une tendance lourde, beaucoup mieux attestée, dont on pouvait prévoir la croissance. La première conduisait à un modèle formellement rationnel d'orientation (forcément autoritaire) des élèves et de refonte des organigrammes disciplinaires, modèle doté de variables calculables, mais dont les paramètres restaient indéfinis, puisqu'il était fondé par une anticipation volontariste des besoins à venir du système productif en main-d'œuvre "qualifiée " et "spécialisée "; la seconde demande, pouvait donner lieu à observations réitérés et se trouvait formulée dans les faits par les familles et les usagers à partir de leurs propres ressources et aspirations. Elle exprimait les valeurs d'une autre rationalité, celle toute individuelle d'une stratégie généralisée de mobilité sociale, appelant une ouverture de l'Ecole à des publics croissant. En France, le pouvoir politique passait, sous l'influence des calculs du Plan, entre 1960 et 1968 de l'utopie ultra-démocratique du plan LangevinWallon à la stratégie restrictive du plan Fouchet, visant à arrêter la montée des effectifs scolarisés après le $\mathrm{Bac}$, qui fut pour beaucoup dans les révoltes étudiantes contre toute idée de sélection à l'entrée des universités en 1968, comme ensuite. L'ère thatchérienne inversera plus tard les attendus des deux prospectives du développement des universités et de la recherche, en attendant les prochains chassécroisés des politiques françaises et anglaises de l'éducation.

\section{2/. Méthodes}

3.8 Dès 1967, nous nous sommes imposé un recyclage méthodologique de statistique générale, et plus particulièrement en statistique non-paramétrique, comme ce fut, je suppose, le cas pour la plupart des historiens, des juristes, des philosophes devenus sociologues qui, avant la création de la licence de sociologie venaient nécessairement d'une autre culture disciplinaire Je me souviens que mon enseignement dans cette nouvelle licence (puis pour la thèse de $3^{\text {ème }}$ cycle nouvellement créée ) répondait à une urgence dans la formation des étudiants qui, jusque là, n'avaient connu de la sociologie que quelques "idées " sociologiques pour les besoins du certificat de "Morale et sociologie " dans la licence de philosophie Apprendre aux étudiants le maniement logiquement correct du langage des "variables" sans pour autant oublier tout ce que l'interprétation sociologique des résultats d'un " croisement multivarié " doit à leur " contexte", toujours "singulier" dans une science historique où on ne rencontre jamais, dans une comparaison, la réitération d'un système de co-occurrences "toutes choses étant égales par ailleurs". Je me souviens de la difficulté à bien faire entendre - par-delà l'opposition paresseuse entre "sociologie quantitative " et "sociologie qualitative " - l'enseignement méthodologique, 
à la fois de formation et d'auto-formation, que j'ai donné là-dessus, en même temps que sur le lexique des théories sociologiques, pendant plusieurs années, comme assistant de Raymond Aron à la Sorbonne ${ }^{[5]}$.

3.9 L'apport des méthodes statistiques aux travaux du Centre n'était pas de simple illustration ou de fairevaloir comme il arrivait fréquemment à cette époque ; il s'agissait bien dans tous ces travaux, comme on l'a vu plus tard de compétences et de techniques statistiques d'analyse des données dont l'emploi était commandé par l'intention sociologique des enquêtes sur l'éducation. Quand nous nous associâmes en 1961 Bourdieu collaborait déjà, depuis 1958 en Algérie, avec des statisticiens et démographes comme Alain Darbel ${ }^{[6]}$ dans le cadre d'une association de recherches (l'ARDES) financée par la Caisse de développement de l'Algérie. Cette collaboration de Bourdieu avec plusieurs divisions et administrateurs de I'INSEE continuera longtemps et s'étoffera en particulier dans les années 70, à partir de son enseignement à l'ENSAE qui éveilla dans les nouvelles générations de statisticiens et d'économistes un intérêt pour le contenu sociologique et historique de leurs opérations et objets formels. Inflexion sociologique observable, entre autres renouvellements du "métier de statisticien " (Michel Volle, 1981), chez de jeunes statisticiens comme Alain Desrosières ou Laurent Thévenot venus travailler au Centre, qui, à partir du réexamen des enquêtes nationales des années 70 sur les ménages, contribuèrent à la refonte de la nomenclature des recensements et enquêtes de l'INSEE. J'ai côtoyé dans la discussion ou l'enseignement ces chercheurs, plus particulièrement Alain Darbel et, plus tard, au Service des statistiques de l'Education Nationale, Claude Seibel puis Claude Thélot, rencontré lorsque j'enseignais la sociologie à l'Université de Nantes, qui, dans le traitement des données que rassemblait les Services de ce Ministère comme dans l'orientation des enquêtes qu'ils lancèrent étaient l'un et l'autre autant sociologues que statisticiens ${ }^{[7]}$.

3.10 Personnellement je m'étais surtout familiarisé avec les conséquences méthodologiques qu'il convenait de tirer du calcul des probabilités dans l'interprétation sociologique des résultats d'une enquête quantitative, selon les contraintes que l'on imposait à ce calcul en fonction de l'objectif attendu d'un traitement de mesures sur des objets historiques, et cela grâce à la distinction clairement établie par Desabie entre "statistique analytique " et "statistique descriptive " (Desabie 1966). C'est avec un autre responsable de l'INSEE, Alain Darbel, qu'avaient été discutées en 1963 les formes sociologiques d'une mesure " adéquate " (au double sens qu'exige Max Weber) de l'inégalité des chances socialement conditionnées de réussite scolaire. Darbel nous proposa un modèle opératoire du maniement des probabilités adapté aux exigences des raisonnements comparatifs de la sociologie, qui a permis de construire, pour Les Héritiers, un algorithme du calcul des chances sociales d'accéder à l'Université propres aux diverses catégories d'étudiants, puis des chances de ces mêmes catégories de s'y trouver réparties, dans des proportions différentes entre les facultés d'avant 1968 : à l'époque droit, médecine, sciences ou lettres et sciences humaines. L'algorithme favorisait sans distorsions ni biais la comparaison sociologique, entre domaines et périodes, des "probabilités objectives " d'accès à l'Université comme des "probabilités conditionnelles" de se retrouver dans une filière donnée, une fois supposée acquise la présence d'une catégorie d'étudiants dans l'enseignement supérieur. Ainsi pouvait-on croiser les probabilités a priori, pour une cohorte quelconque, de réussir à un niveau ou dans une filière d'études, en fonction du statut du père ou de la mère, comme du sexe, de la résidence, du diplôme antérieur ou de toutes ces caractéristiques simultanément, en se basant sur les chiffres réels de recensements de ces catégories à la naissance des individus ou au départ d'un processus, en même temps que sur les chiffres tout aussi accessibles d'inscription, d'échec ou de réussite à l'université, que l'algorithme mettait ainsi en relations dans un bilan dressé a posteriori. De même, je trouvai plus tard dans "l'analyse factorielle de correspondances ", telle que Benzécri en systématisa les principes probabilistes, une technique de prospection des corrélations prises deux à deux entre toutes les variables d'un corpus de données ${ }^{[8]}(18)$, qui se prêtait, mieux que le traitement des données par une succession de « tableaux croisés ", à l'interprétation sociologique d'un écheveau d'interdépendances multiples

3.11 À la fin des années 60 , le " calcul des chances ", effectué en termes de probabilités mesurées en divers points d'une trajectoire, a fait changer le regard de la société sur les inégalités culturelles. Dans les années 70 , la rencontre entre le raisonnement sociologique portant sur les procédures du traitement quantitatif des données et l'affinement probabiliste du calcul statistique a renouvelé radicalement les programmes d'analyse de la démocratisation de l'école et de la culture, comme il le faisait depuis longtemps dans l'étude d'autres processus temporels, par exemple sur des tables de mobilité sociale ou d'évolution de l'endogamie et de l'hétérogamie entre les différentes catégories sociales d'une même société, tous calculs qui pondèrent les "chances » de changer de catégorie dont sont dotés les descendants de chaque catégorie en fonction de leur poids dans la population totale; ou, dit en langage technique, en fonction des " contraintes de marge » propres à tout tableau comparatif de changements intergénérationnels.

3.12 La tache d'une sociologie de la démocratisation ne s'épuise pas, en effet, dans la description et la mesure de la composition sociale des publics fréquentant les institutions culturelles, officielles, officieuses ou marginales. La toute simple démultiplication de la « règle de trois »- pont-aux ânes de l'apprentissage statistique, pas toujours facile à franchir comme on le voit dans la résistance des étudiants en sociologie à la manipulation correcte des pourcentages en lignes ou colonnes, c'est-à-dire à la comparaison des "fréquences relatives " rapportées à une même base pour calibrer les quantités comparées - a vite fait déceler la déformation des probabilités réelles d'accès à une pratique ou une performance qui se glisse dans la plupart des "tableaux croisés » construits indépendamment de toute réflexion sur leur diachronie et sur le volume initial des cohortes prises en compte. Il y a un biais de calcul dans les comparaisons de séries, lorsqu'on les coupe de leur contexte démographique, en se bornant à suivre l'évolution du nombre des représentants des différentes catégories d'une population (dans une institution donnée ou à un moment donné du cursus), alors que le poids démographique de ces catégories est fort inégal dans la population de référence et qu'il a lui-même évolué au fil des générations durant la période de comparaison. Il est particulièrement trompeur pour l'époque de transformation rapide de la population active dans les économies capitalistes des deux derniers siècles : exode rural d'abord, puis basculement en deux 
générations des effectifs du secteur secondaire dans le secteur tertiaire, avec apport significatif des mains-d'œuvre immigrées dans les métiers les moins qualifiés.

3.13 « II n'y a que 6\% de fils et filles d'ouvriers à l'Université, contre 30\% d'enfants de cadres supérieurs, professions libérales et gros patrons » s'exclamait-on en 1963 dans les syndicats étudiants ou enseignants de gauche, qui croyaient prendre ainsi la mesure exacte de l'inégalité des chances de réussir à l'Ecole pour ces catégories socio-professionnelles situées aux deux bouts de l'éventail, en se fiant à l'impression trompeuse qu'au vu des chiffres d'inscription des étudiants et étudiantes de différentes origines sociales, en cette année universitaire 61-62, la classe ouvrière n'avait que 5 fois moins de chances que les catégories les plus privilégiées de voir ses enfants entrer à l'Université. Si, au contraire on calcule les chances d'accès et de réussite à l'Ecole en fonction de l'origine sociale selon le modèle probabiliste conçu par Alain Darbel [9] (19) qui rapporte, à partir de données disponibles à l'INSEE, les chiffres d'inscrits de la catégorie la moins représentée, à un niveau ou dans une filière d'études, au fait qu'elle était alors et de loin, la plus nombreuse dans la population active vingt ans auparavant - et l'inverse pour la catégorie la plus représentée à l'Université - on aperçoit que la probabilité d'entrée à l'université selon la catégorie sociale de naissance était, en 61-62, de 2,4 chances sur 100 pour les fils et filles d'ouvriers et de 58 chances sur 100 pour la catégorie des « héritiers », soit dans un rapport de 1 à 30 entre les chances de ces catégories extrêmes ${ }^{[10]}$.

3.14 L'expression d'« égalité de chances » n'est pas nouvelle dans les raisonnements politiques et éthiques sur les inégalités sociales, mais on s'avisait rarement qu'on ne les mesurait pas vraiment en termes de «probabilités objectives " (ou de «probabilités conditionnelles ") d'accès à un bien ou une position dans la comparaison entre les différentes catégories de départ, définies par des critères qui étaient pourtant disponibles dans les recensements. II me semble que, depuis les années 70, l'habitude s'est prise chez les sociologues de raisonner et de calculer en termes de probabilités pondérées (a priori ou a posteriori selon les données utilisées et la question posée) sur les inégalités sociales de toute nature, c'est-à-dire sur les chances de se retrouver ou non usager d'un service ou possesseur d'un bien (comme dans le calcul devenu classique des chances scolaires en fonction de la composition sociale d'une cohorte démographique née vingt ans auparavant). On caractérise alors, non plus la simple composition sociale d'un public (par les \% de chaque catégorie sociale qu'il contient) mais les chances pour chacun des membres d'une catégorie de départ de se retrouver dans une pratique et non dans une autre ou à un niveau plus ou moins élevé d'intensité de cette pratique (par les \% de pratiquants que contient en chacun de ces cas la catégorie de départ considérée) ${ }^{[11]}$. Bref, on se rapproche, par le calcul des chances, de la perception sociale que les individus ont de « ce qui se passe » dans leur environnement proche (donc accessible à l'enquête de proximité) ; c'est-à-dire qu'on saisit la base des représentations du lien vécu entre le « réalisable » et le « désirable », qui les motivent ou les démotivent en leur faisant apparaître les futurs possibles comme plus ou moins probables en fonction de leur expérience quotidienne. C'est cette variation sociale de «l'espérance subjective » d'un futur particulier - produit d'expériences précoces et réitérées - qui explique les « attentes », « vocations », « aspirations », les consentements aux « coûts » d'un apprentissage, ou le dégoût de l'effort scolaire, l'adoption ou le refus d'une patience préalables, bien plutôt que la fatalité ou le déterminisme opaque d'un « destin social » de classe ou de sexe qui se réaliserait mystérieusement chez chaque individu par une « intériorisation » directe des « probabilités objectives », connues des seuls statisticiens qui les calculent a posteriori sur des recensements ou des échantillons représentatifs.

\section{3) Apports et résultats}

3.15 Les apports de nos travaux ne consistaient pas seulement dans des chiffrages de fréquences faisant preuve de relations causales entre l'origine sociale et la réussite scolaire ou l'insertion professionnelle, mais aussi dans le sens élargi que prenaient ces constats lorsqu'on les mettait en rapports avec les analyses d'autres sociologues dont nous découvrions la pertinence descriptive et explicative, pendant que nous écrivions Les Héritiers : Richard Hoggart d'abord, dont j'ai parlé, avec ses descriptions de la trajectoire d'un " boursier" parti de Leeds dans le Nord-est industriel de l'Angleterre et surtout ses analyses de l'attention captive, mais en même temps flottante et "distraite " que les classes populaires anglaises réservaient aux produits de l'édition et de la culture de masse (" savoir en prendre et en laisser ") ${ }^{[12]}$; ou Basil Bernstein dont la théorie du rôle que jouaient le "code restreint " et le "code généralisé " dans la socialisation verbale, affective et intellectuelle des enfants des classes populaires et de ceux des classes moyennes [13], rencontrait les résultats de nos enquêtes sur le langage scolaire et les malentendus de la relation pédagogique dont nous décrivions les dysfonctionnements dans la communication entre enseignants et enseignés [14]

3.16 Les résultats les plus déterminants de nos enquêtes consistaient alors dans l'étayage empirique de quelques bases théoriques dont nous commencions à être assurés, Bourdieu et moi, à la fin des années 60 : (a) la définition durkheimienne de "l'institution" comme cadre de la durabilité des représentations et des attitudes; (b) la définition par Marx de la base économique et de la portée idéologique des différences et conflits de classe ; et enfin (c) le rôle actif que jouent les " représentations " de la légitimité qu'accordent les acteurs sociaux à un " pouvoir d'ordonner", c'est-à-dire de cette force symbolique de normativité que Weber avait minutieusement décrite, à travers plusieurs civilisations, dans différents " ordres " (juridique, éducatif, religieux ou politique) - ce qui nous incita après avoir éprouvé sa puissance interprétative dans le corpus wébérien à transposer à "l'ordre culturel "le concept de "légitimité " prêté aux différents " ordres sociaux" analysés par Weber.

3.17 Les résultats de cette sociologie de l'éducation - on distinguera plus bas les apports qui ont survécu ou prospéré dans la recherche sociologique et ceux qui se sont le plus facilement dégradés en stéréotypes dans les polémiques entre agitateurs intellectuels, chez les militants utilisateurs de cautions scientifiques, 
dans les conversations dilettante du grand public ou dans les débats tourbillonnants des médias. consistaient au début des années 70 en un certain nombre de constats appuyés à la fois sur la mesure, l'interview, l'analyse de documents ou d'archives et l'observation de terrain. Précisant le rôle de l'Ecole, institution elle-même auto-reproductrice de ses valeurs et hiérarchies constitutives, au cœur des mécanismes de la reproduction socio-culturelle et idéologique, observables dans toutes les sociétés culturellement stratifiées, nos recherches sur l'éducation dégageaient au moins :

- des instruments homogènes de mesure de l'ordre de grandeur des inégalités devant l'Ecole et la culture scolaire ou devant d'autres pratiques culturelles, selon l'origine sociale, le sexe, l'habitat, le passé scolaire comme selon les catégorisations obtenues par le croisement de plusieurs de ces variables;

- des données sur leur évolution historique, qui devenait sociologiquement mieux intelligible quand on la restituait au contexte de l'histoire sociale de l'Ecole et des transformations de la stratification sociale qui l'accompagnaient ;

- le rôle "compréhensible " du "capital culturel " hérité des parents et de leur environnement proche dans la réussite scolaire lorsqu'on l'analyse comme un système de "ressources" symboliques ; de même pour la reconnaissance de la "légitimité " de l'Ecole et de ses sanctions par ses usagers. Symétriquement, il y a toujours lieu de décrire les conditions de la "délégitimation " du pouvoir d'être obéi pour expliquer une "crise", surtout dans une institution comme l'École dont "I'autorité " ne peut-moins que tout autre-s'appuyer sur la seule force nue.

- le rôle efficient du "capital culturel " dans l'orientation et l'intensité des transactions sociales, tant à l'Ecole où il conditionne le succès aux examens qu'ultérieurement dans le déroulement des carrières professionnelles où il se transforme, pour une part, en "capital social " (prestige, influence) et, pour une autre, en "capital économique " (revenus, patrimoine);

- Enfin, une certaine reconfiguration des formes de l'administration de la preuve dans le travail d'analyse sociologique fondant ces ouvrages qui associaient intimement plusieurs méthodes de description et d'explication : celles qui étaient les plus liées au traitement statistique des données sociales comme les formes les plus dépendantes du récit et de la comparaison historique. Cette synthèse, implicite dans les premiers ouvrages de sociologie de l'éducation se formula explicitement, à partir de 1968, dans Le métier de sociologue qui avait été conçu pour favoriser un changement de posture épistémologique chez les jeunes chercheurs que nous voulions former ${ }^{[15]}$. Mais Le métier était tout aussi chargé de mésinterprétations possibles par le lecteur selon les domaines et les disciplines où il appliquerait cette leçon d'épistémologie bachelardienne ; et cela d'autant plus facilement que les auteurs traitaient, dans leur langage conceptuel, leurs désaccords théoriques, par des compromis sur les points où, de fait, ils divergeaient sans le laisser apercevoir par leurs formulations négociées - comme par exemple sur le rôle exclusif accordé à la "sociologie de la sociologie " tant dans la critique que dans la réorientation de l'épistémologie des sciences sociales. La divergence entre les deux auteurs, après 1972, trouve là son origine et explique le style argumentatif de plus en plus différent de leurs ouvrages ultérieurs, sur fond d'une sociologie de la socialisation et de l'acculturation restée assez proche ${ }^{[16]}$.

\section{LA RECEPTION DES HÉRITIERS ET DE LA REPRODUCTION, LORS DE LA PUBLICATION ET JUSQU'AUX ANNÉES 80}

\section{1/. Avant 1968}

4.1 Les Héritiers ont été écrits dans une période d'effervescence autour d'une réforme de l'Ecole que tous les groupes sociaux (usagers ou responsables) considéraient comme urgente et inéluctable mais qui ne s'accordaient que dans leur intention polémique alimentée par des attendus éclatés, parfois même parfaitement contradictoires. Ayant assisté à des colloques, comme ceux de Caen ou d'Amiens qui brassaient cette critique de plus en plus véhémente et bariolée de l'Université traditionnelle, l'unanimité négative qui s'y fit jour, en même temps que dans les tribunes de presse, m'évoqua souvent l'unanimité lyrique des assemblées révolutionnaires où le "baiser Lamourette " des députés de la Constituante resta sans lendemain. L'alliance, fusionnelle dans l'opposition, entre le réformisme de rénovateurs politiques ou technocrates, des programmes de chercheurs et des lignes politiques de partis, celui des revendications catégorielles de professeurs et d'étudiants, celui des analyses d'universitaires ou de savants reconnus inquiets de la sclérose de l'institution, et des analyses de clan ou de ressentiment, celui des projets d'intellectuels révolutionnaires ou utopiques dont le radicalisme s'étendait jusqu'aux disciples d'Ivan Illitch prônant une " désinstitutionalisation " radicale de l'Ecole, vola évidemment en éclats en mai 68, dès l'occupation de la Sorbonne et la diffusion de masse d'un militantisme étudiant qui se refusait aux examens. Mais, jusqu'aux jours de l'explosion, toutes les critiques de l'Université traditionnelle étaient adoptées allégrement par l'opinion en mouvement. Je me souviens qu'avant la parution du livre, l'argument que nous présentions le plus souvent dans des débats, face aux syndicats étudiants ou révolutionnaires et aux professeurs réformistes, aux planificateurs technocrates comme aux pédagogues - celui de la critique d'une "pédagogie de la non-pédagogie " des enseignants du Supérieur qui contribuaient par leur cécité magistrale aux malentendus et à l'échec scolaires - était adopté sans difficultés par l'auditoire, les arguments critiques étant toujours bons à cumuler pour une opposition ou une contestation.

\section{2/. De la parution des Héritiers, jusqu'à La reproduction et au-delà}

4.2 Les réactions aux Héritiers et à La reproduction ont été multiples et surtout changeantes au gré des crises scolaires surtout après 1970, qu'il s'agisse de la captation politique des arguments sociologiques issus de ces deux ouvrages en fonction du renouvellement des enjeux et des publics ou des débats et critiques (méthodologiques ou théoriques) parmi les sociologues de profession qui se sont intéressés à leur démarche de preuve ou leur usage de la statistique. 
4.3 Pour les stéréotypes, comme il est après tout de règle en toutes les réceptions d'ouvrages lus un peu au-delà du cercle des pairs et des spécialistes j'ai surtout remarqué la rapide succession des

"renversements du pour au contre" dans les opinions militantes après 1968. Dans les sectes gauchistes, comme chez les militants du PC, selon leurs changements de ligne ou d'humeur. Les collègues réformistes qui avaient apprécié le projet de substituer à l'absence de pédagogie, à quoi se réduisait la pédagogie traditionnelle à l'Université, une " pédagogie rationnelle " suggérée dans Les héritiers pour une réforme de l'Ecole qui voudrait appliquer la connaissance sociologique au rattrapage des handicaps sociaux et culturels, confondirent vite cette redéfinition des fonctions de l'Ecole avec l'impasse idéologique d'une "Université critique " qu'ils refusaient. D'autres publics y avaient lu autre chose; et en somme les enseignants les plus conservateurs sont les seuls à n'avoir jamais changé dans leur détestation de deux ouvrages irrémédiablement lié dans leur mémoire au choc de 1968. Plus généralement, beaucoup de critiques formulées sur le moment par des sociologues d'enquête cachaient mal que leurs objections à nos preuves procédaient d'un désaccord avec la ligne de politique pédagogique qu'ils nous prêtaient par référence à la tournure que prenaient les évènements universitaires au fil des années 70. Ouvrages "pousse-au-crime" : tel resta, chez beaucoup de nos collègues, exaspérés par le déclin de "l'autorité " des enseignants dans les décennies suivantes, le diagnostic dominant.

4.4 A ma grande surprise, les courriers que je reçus dans les mois qui suivirent la parution des Héritiers émanaient principalement de "boursiers " anonymes ou d'intellectuels que j'avais connus au cours de leurs études, parfaitement rodés à la culture scolaire et qui pourtant, les uns et les autres, vivaient comme une "libération " affective, leur soulagement à voir décrite et neutralisée la banalité sociologique d'une origine sociale modeste qu'ils avaient vécue en ces années là dans la " honte " ou le malaise suscités en eux par les humbles professions du père ou de la mère, souvent dissimulées à leur condisciples. En revanche les articles professionnels ou les comptes-rendus de l'ouvrage que je lus entre 1964 et 1968, lui reprochaient surtout d'introduire une virulence politique déplacée dans la sociologie universitaire : plusieurs sociologues traquaient l'erreur de pourcentage dans les tableaux statistiques et les graphiques ; certains plus audacieux voulurent prouver que nous nous trompions de "variable lourde" : c'était le travail hors des études, non l'héritage culturel, qui handicapait la réussite des étudiants originaires de familles défavorisées. Le problème de sociologie de la culture était clos par un retour pur et simple à l'explication strictement économique que préféraient les politiques et les syndicalistes de gauche. Comme les deux variables considérées étaient en colinéarité, il suffisait évidemment de changer de "variable indépendante " pour interpréter différemment les mêmes valeurs numériques. Mais, en gros, le débat sur la valeur scientifique des conclusions des Héritiers (et plus encore de La reproduction) a toujours été plus idéologique que technique.

4.5 Les uns y voyaient tiédeur réformiste, les autres dénigrement des contenus scientifiques de la culture universitaire. Fluctuations et contradictions s'étaient manifestées dès avant 1968. Je les ai alors observés dans des forums et des rencontres avec professeurs, étudiants, syndicalistes, administrateurs et journalistes, où je présentais nos premiers résultats d'enquête. L'UNEF, par exemple, qui utilisait volontiers nos constats d'inégalité sociale dans les études pour y fonder sa revendication d'un " pré-salaire pour tous ", seul capable d'émanciper toutes les catégories d'étudiants de la dépendance familiale; restait sourde à une sociologie des variations du lien constaté entre les budgets des étudiants et ceux de leurs familles. Plus utopiques encore le refus de cette sociologie avait été inauguré avant 1968 par les "situationnistes " dont le Manifeste ${ }^{[17]}$ épinglait les duettistes "Bourderon et Passedieu " qui " dans leur enquête Les héritiers : les étudiants et la culture, restent désarmés devant les quelques vérités partielles qu'ils ont fini par prouver. Malgré leur volonté bonne, ils retombent dans une morale de professeurs, dans l'inévitable éthique kantienne d'une démocratisation (...) du système d'enseignement (..) par l'enseignement du système ". Bref, les " nouveaux mandarins "- comme diront Lapassade et la plupart des courants du "mouvement étudiant " à partir de 1969- sont plus pervers que les anciens, par le soutien intellectuel que leur relativisme apporte à l'ordre capitaliste : entre bien d'autres documents d'archives, il suffit de lire un numéro de Labo-contestation $\left(n^{\circ} 6,1972\right)$ qui présente sa sociologie humoristique de la guéguerre entre professeurs de sociologie, où figurent, également caricaturés, à côté de mandarins traditionalistes ou technocrates (Aron l'Ancêtre, Blanc Boudin ou Le Cuistot), les faux amis d'une émancipation de la pensée critique : Toutou le Grand manitou, Babar-Altuzère et Bourdon-Passérieux.

4.6 Même chez les sociologues les plus soucieux d'objectivité, la résistance était forte. Je me souviens, par exemple, des remarques critiques et parfois acerbes de Raymond Aron qui subodorait dans les constats empiriques de privation ou de dépossession chiffrés par Les Héritiers la présences sournoise du concept $d$ '“ aliénation " pour lui indistinctement hégélien, feuerbachien et sartrien. Je vis dans les conversations avec lui qui suivirent le succès de ce petit ouvrage, lorsque je le croisais le matin au Centre - lui, dictant par téléphone sans jamais se reprendre un éditorial au Figaro, moi préparant laborieusement mon enseignement de l'après-midi sur Weber - qu'il opposait avec conviction à cet ouvrage deux arguments venus de Pareto pour affirmer son scepticisme de sociologue tant envers la stratégie irrationnelle d'une égalisation trop accentuée des chances scolaires et sociales au départ dans la vie, arguant de l'invivabilité psychologique que produirait une méritocratie intégrale où les ratés ne pourraient plus s'en prendre qu'à eux-mêmes et à leur médiocrité génétique de leur échec social. II tenait moins au premier argument parétien, devenu de fait intenable avec les découvertes de la génétique moderne et le développement de la statistique d'analyse de vastes agrégats statistiques, qu'au second qui attirait l'attention sur les " coûts sociaux " de la discontinuité des transmissions culturelles s'opérant dans les lignées familiales tant pour les individus déstabilisés par leur mobilité ascendante que pour les souffrances des individus déclassés dans une lignée: "A quoi bon amener de loin et à grands frais des parvenus culturels aux postes de responsabilité quand les héritiers de l'élite, pour qui les familles aisées faisaient l'essentiel du travail et de la dépense, suffisaient à la reproduction de l'équilibre social ? ". L'argument transposait sociologiquement et économiquement le raisonnement conservateur de Pareto qui tirait, lui, du phénomène de la "circulation des élites "et de l'essorage des talents nés dans des classes populaires par l'ascension sociale la conclusion biologique qu'il s'opérait continûment un appauvrissement du patrimoine 
génétique des masses. Pour tout dire, il se trouve que ces argumentations aroniennes m'amenèrent à lire plus attentivement le double message scientifique de Pareto économiste et sociologue, à qui depuis j'ai toujours rendu justice, nonobstant son épistémologie crûment positiviste, comme architecte d'un des systèmes les plus ambitieux des sciences sociales, qui englobe l'économie dans la sociologie en associant explicitement les bases du Cours d'économie politique à celles du Traité de sociologie générale, pour faire ressortir la spécificité de "l'équilibre social " inaccessible au calcul formel qui lui avait permis de maîtriser "l'équilibre économique " dans une théorie mathématisée. Ainsi conçue comme une science historique, la sociologie ouvre plus largement l'explication des " dérivations "que ne le fait l'économisme de l'explication des idéologies chez Marx.

4.7 D'autres chercheurs en sciences exactes, mathématiques ou biologiques, dont les plus notoires étaient associés au mouvement des universitaires réformistes qui précède 1968 ou s'y diversifie, me révélèrent une autre dimension du malentendu entre disciplines lorsqu'elles réfléchissent " théoriquement "en s'éloignant de leurs méthodologies constitutives. Je me souviens d'un débat public juste après 68 (dans la salle Dussane de l'ENS) où le mathématicien Laurent Schwartz voulait nous manifester son accord à propos des débats didactiques sur la pédagogie (universitaire) de la non-pédagogie en décrivant hyperboliquement le rôle que "l'élégance " de la démonstration jouait, selon lui, chez trop d'enseignants en mathématiques, rôle plus important, disait-il, que celui du biais "littéraire " dans l'enseignement facultés de lettres. Je découvris d'ailleurs dans sa plaidoirie anti-élitiste mon premier exemple d'un "schème " d'argumentation - logiquement pervers et très fréquent dans la rhétorique de colloque : "Vous avez tellement raison qu'au bout du compte vous avez tort "- ce qui permet de débuter par l'affirmation que l'on va "prolonger l'intervention de l'orateur précédent" pour en venir à une invalidation (enquête ou pas enquête) du fondement empirique de sa thèse. Schwartz disait en somme "le phénomène que vous avez observé est certain, mais vous avez tort de le lier préférentiellement aux techniques de l'enseignement dans les facultés des lettres, puisque je crois qu'il est encore plus étroitement lié à la pédagogie des sciences exactes. Dissymétrie des méthodologies du constat : nos enquêtes empiriques portaient exclusivement sur l'enseignement des sciences de l'homme et de la philosophie, alors qu'il n'en existait pas encore sur la pédagogie et la morphologie des filières scientifiques. Plus tard, les enquêtes sur l'enseignement scientifique devaient montrer que le "plus " n'était fondé que sur des impressions d'universitaire spécialisé passionné par sa pratique d'enseignant et d'inventeur.

\section{III/. LA DIFFUSION DES APPORTS SOCIOLOGIQUES DES HERITIERS ET DE LA REPRODUCTION, DISTINGUÉE DE LEUR INFLUENCE COMME "STÉRÉOTYPES " A SUCCĖS}

5.1 La distinction est évidemment difficile lorsqu'il s'agit de l'influence de travaux immédiatement lus et mis en conversation dans le grand public intellectuel comme ceux dont il est question ici, qui n'ont jamais été des best-sellers, mais jusqu'ici, pour Les Héritiers et La reproduction, des long-sellers. Lorsque les comptes-rendus de spécialistes s'affrontent en vase clos, lorsque la répercussion dans les médias aiguise et entretient les polémiques, lorsque l'actualité politique et les péripéties de la vie universitaire incitent à forcer le trait, le contenu scientifique des affirmations issues de la recherche ne peut plus guère être séparé par quelque public que ce soit des réconforts ou des indignations qu'il y puise. Mais ce phénomène n'etait pas plus accentué, il me semble, à propos de notre sociologie de l'éducation qu'à propos de tout autre recherche dans une science sociale, ainsi démarquée par son audience plus large, du public habituel des sciences exactes, y compris dans les vulgarisations. Dès lors qu'il suggère un sens inattendu ou trop attendu par ses contemporains, un message scientifique est toujours moins entendu dans ses assertions dénotatives que dans ses connotations amalgamatiques. 1)

\section{Réceptions liées à la conjoncture, impliquant contre-sens et stéréotypisations outrancières}

5.2 On ne peut, bien sûr, les énumérer ici : elles fourniraient un matériel abondant pour l'étude des " pactes de lecture "des œuvres scientifiques en fonction des " attentes " des publics, pactes plus "sauvages " encore que les pactes de réception qu'étudie la sociologie de la réception des œuvres littéraires ou artistiques : j'en trouve à foison dans mes courriers et mes dossiers de coupures de presse. Disons qu'elles se caractérisent toujours:

- Par une sélection qui radicalise jusqu'à la caricature ou l'inversion, en les séparant de leurs moyens de preuve, les constats ou interprétations les plus solidement argumentés : utilisations gauchistes de quelques phrases de La reproduction auxquelles je me heurtais à Vincennes, récupération par la traduction dans la langue de bois des partis, escalades catégorielles dans les citations syndicales ou contestataires, lectures dans le style des " notes de synthèses " pour les planificateurs technocratiques en quête de "stratégies " efficaces capables de les démarquer du "fonctionnalisme du pire " prêté à toute description sociologique des obstacles culturels à la rationalisation formelle. L'accusation de " marxisme ranci " qu'adressait Peyrefitte à La reproduction appartient au même registre de lecture que celui des indignations professorales contre la chienlit qu'encourageaient nos textes, ou que celui des révoltes étudiantes contre toutes formes d'AuP (autorité pédagogique), d'Ap (action pédagogique), contre tous les "ordres sociaux " agglomérés sous l'appellation maléfique de "système".

- Et surtout par des chassés-croisés rapides et peu prévisibles dans la reprise des thèmes qu'empruntaient à notre sociologie de l'éducation les textes à succès de pamphlétaires et d'agitateurs. Le la de la contestation de "toute autorité " conçue comme répressive, de tout système d'organisation ou de règles, a plutôt été donné dans le grand public, après 70, par ces rewriting de propagande que par la lecture de nos propres ouvrages.

5.3 Mais je crois que c'est là un phénomène banal dans les rencontres entre discursivités qui reposent sur des modes argumentatifs différents. Je crois en tout cas que c'est toujours sur des lectures sélectives que repose la réception des messages scientifiques ou littéraires par le grand public intellectuel. " L'horizon 
d'attentes " des publics flottants est d'autant plus facilement comblé que les attentes par l'auteur d'un lecteur taillé à la mesure de son message sont plus éloignées des attentes de lecteurs prêts à adhérer à leurs propres attentes sans lire vraiment le détail du message. "C'est par le malentendu universel, écrivait Baudelaire, que tout le monde s'accorde. Car si, par malheur, on se comprenait on ne pourrait jamais s'accorder. "(Beaudelaire 1887). Si ce principe de "pragmatique " de la conversation est trop poétique pour être vrai universellement, il fournit au moins un indicateur de la frontière entre communication scientifique et communication quotidienne; mais il révèle aussi les conditions et les terrains de leurs interférences.

\section{2). Les concepts scientifiques et leur influence}

5.4 Je ne crois pas pour autant que les lectures ou usages caricaturaux des ouvrages de sociologie de l'éducation co-signés par Bourdieu et moi aient empêché leur lecture critique par les sociologues qui nous suivirent ou leur influence sur les recherches sociologiques ultérieures. D'ailleurs, les sociologues qui les ont, sur le moment, un peu trop brutalement dénoncés, décalqués ou exagérés oublient aujourd'hui assez spontanément l'usage qu'ils font eux-mêmes des concepts descriptifs et des démarches de preuve élaborés dans ces ouvrages ; je crois que c'est tout simplement parce qu'ils les utilisent - et à juste titre comme un bien désormais commun de l'outillage sociologique de leurs conceptualisations et de leurs enquêtes. II me semble que quatre schèmes d'explication ou de méthode apparus dans ces premiers ouvrages sont aujourd'hui devenus d'usage courant dans les recherches sociologiques, indépendamment des affiliations d'école.

5.5 a). D'abord le rôle de "l'héritage culturel " est généralement accepté comme le premier facteur explicatif à tester dans les enquêtes sur la réussite scolaire, professionnelle ou sociale comme sur les choix économiques, culturels, politiques ou artistiques opérés par des individus ou groupes sociaux dotés de ressources symboliques différentes. Je crois même que c'est cette formulation d'une intelligibilité rationnellement "compréhensible" par la signification historique ainsi conférée aux continuités intergénérationnelles qui explique, sur pièces et dans la diversité des "cas " ou séquences singulières, la plupart des régularités sociales. En tout cas elle a été la première à être reprise par d'autres sociologues au moins comme expression commode, pour démarquer l'explication proprement sociologique de la reproduction sociale des explications par l'hérédité des patrimoines génétiques. Je me souviens par exemple de la succession et de l'évolution "théorique "des enquêtes et publications de l'INED sur la réussite et l'échec scolaires. J'avais, entre 1962 et 1963, vers la fin de la première vague d'enquêtes qui faisaient la matière des Héritiers, rencontré Paul Clerc à l'INED - institution avec laquelle j'avais plus de contacts, ceux de Bourdieu étant plus étroits avec l'INSEE - pour m'assurer que les enquêtes en cours dans cette Institut sur la " déperdition scolaire " enregistrée au fil des études, en fonction de l'origine sociale, n'étaient pas les mêmes ou trop proches des résultats que nous allions publier. En échangeant quelques informations sur la structure et les objectifs des enquêtes menées de part et d'autre, je fus rassuré, au cas où j'aurais eu quelques craintes sur l'originalité (ou la primauté) des nôtres concernant les facteurs sociaux de la réussite des étudiants dans les universités françaises, puisque les cohortes suivies par les enquêtes successives de l'INED portaient au départ sur des élèves de CM2 d'établissements primaires, ensuite étendues au secondaire ou à la réussite au Baccalauréat, et que la preuve du rôle joué par l'origine sociale dans la " mortalité scolaire " se trouvait ainsi être d'autant plus forte

méthodologiquement que nous mesurions, dans nos enquêtes, l'influence de la socialisation familiale plus tard dans le cursus - ce que nous n'avions choisi, il est vrai et avec d'autres inconvénients techniques, que pour la commodité de passation des questionnaires par notre réseau d'enquêteurs eux-mêmes professeurs ou étudiants dans l'enseignement supérieur. Mais surtout, je m'aperçus que les hypothèses des chercheurs de l'INED laissaient à cette époque (celle de l'article de 1963 dans Population) l'explication de la "mortalité différentielle" des enfants de différentes origines sociales - qu'ils établissaient par des constats de plus en plus probants à mesure que s'allongeait leur suivi de cohortes - indifféremment ouverte à une interprétation génétique (" héritée " des donnés et des hypothèses d'une enquête de 1944 de la Fondation Alexis Carrel), comme à une interprétation restrictivement économique (alors dominante dans les idéologies de droite ou de gauche du corps enseignant), ou enfin à une interprétation sociologique vague ou dubitative ${ }^{[18]}$. Nous pûmes ainsi relever, avec une certaine satisfaction, l'apparition du terme " d'héritage culturel ", venu probablement des Héritiers, dans l'article de Population co-signé en 1965 par Sauvy et Girard. En ce qui me concerne, il se trouve que c'est la lecture des diatribes de Saint Simon contre la mécanique reproductrice des privilèges de fortune transmis par l'héritage des patrimoine économiques, décrit comme l'obstacle principal à la libération des énergies productives dans une société industrielle, qui m'a suggéré l'extension analogique de la notion d' " héritage " au rôle joué par la transmission d'un patrimoine (ou d'un capital) culturel.

5.6 b). "Le calcul des chances scolaires conçues comme probabilités a priori objectivement attachées à l'appartenance à une catégorie et calculées a posteriori " par la mesure des survivants de cette catégorie de départ à un niveau donné des études. Je ne reviens pas ici sur les différentes logiques et moyens statistiques de mesurer des inégalités sociales. Mais je rappelle ${ }^{[19]}$ les potentialités explicatives de plus en plus sollicitées depuis les premiers usages que nous en avons faits en sociologues tâtonnants, mais qui étaient déjà présentes chez les économistes les plus défiants envers la formalisation intégrale des modèles probabilistes de la décision rationnelle dans l'incertitude totale comme aux frontières de la certitude $^{[20]}$. L'interprétation du rôle des aspirations à la réussite scolaire en fonction des "espérances subjectives ", différentes des "probabilités objectives " de gain ou de perte, que seul connaît le statisticien qui les calcule a posteriori, conduit à rechercher les indices concrets des avenirs probables ou improbables, qui peuvent être connus ou observés par les sujets dans leur environnement proche [21]

5.7 c) La notion de "capital culturel " (scolaire ou mondain, bourgeois ou populaire, symbolique ou politique), souvent critiquée depuis pour sa texture métaphorique, est, et est restée utile, en ce qu'elle suggère par cette analogie des questionnements nouveaux sur les modalités de "l'avoir" et des diverses 
formes de " ressources " par exemple pour interroger les effets de leur articulation avec d'autres composantes, en particulier économiques du "capital social". Il est vain de se demander indéfiniment si, comme tant d'autres termes du vocabulaire sociologique, cette notion est un concept opératoire ou une simple et dangereuse métaphore provocatrice. Utilisée comme matrice d'hypothèses à tester, l'analogie produit, dans les sciences historiques, des occasions de connaissance aussi bien quand elle révèle des parallélismes inattendus que lorsqu'elle disjoncte en révélant des différences inassimilables par son seul pouvoir sémantique ${ }^{[22]}$. C'est par exemple quand on aperçoit la différence insurmontable entre ce qui peut être défini opératoirement comme la "composition organique "d'un capital économique ou le mode de sa transmission par héritage et la nécessaire restructuration de ces notions dans le cas d'un capital culturel, que l'on apprend, par différence, quelque chose de nouveau sur ces deux formes de capital. L'analogie est un détecteur des pertinences comme des non-pertinences.

5.8 d) Remarquons finalement que c'est par leurs descriptions et suggestions pédagogiques que l'influence des Héritiers doit d'avoir perduré, plus longtemps, me semble-il, que celle de La reproduction, bien que les tirages actuels de ces ouvrages jumeaux ne les distinguent guère. Je dois bien conclure que la certitude durkheimienne qu'il existe des relations privilégiées entre sociologie et réformes sociales est toujours vivace. Une sociologie attachée à identifier les régularités locales et à les replacer dans leur contexte ne peut suggérer qu'un réformisme scientifiquement informé : ses mariages d'humeur avec les utopies révolutionnaires ont vite tourné à l'aigre et au divorce. Contestée ou dénoncée pour son angélisme politique - on le voit encore aujourd'hui à propos des ZEP ou des passerelles entre un lycée de banlieue et le recrutement à l'Institut des Sciences Politiques - l'idée d'une " pédagogie rationnelle " qu'esquissaient les analyses des Héritiers est fondée sur le principe que la sociologie des handicaps conduit, lorsqu'elle réussit à dégager quelques bribes de causalité intelligible, à une pédagogie du contre-handicap.

Rencontrant ici Antoine Prost, historien précis de l'éducation en France, qui m'a souvent dit avoir lu ainsi la conclusion des Héritiers, je lui donne volontiers acte que les retombées pédagogiques de La reproduction ne sont pas de la même eau. J'ai expliqué ailleurs, par la sociologie de ses auteurs, ce que le style et l'abrupt des thèses de cet ouvrage, dont Prost fit en son temps une critique assassine et humoristique dans la revue Esprit, devaient, chez Bourdieu et Passeron, à une stratégie de parodie du style universitaire pour mieux imposer la compréhension de ces thèses, dévalorisées comme "primaires ", dans ce même milieu (Passeron 2002:112). Mais je me souviens surtout, en ce qui concerne les liens de notre sociologie de l'éducation avec l'ensemble des enquêtes et des chercheurs travaillant dans le domaine, que, à contrecourant de la perception euphorique de la démocratisation de l'Ecole en France dans les années 70-80, il a conforté par son enquête sur l'évolution de la composition sociale des flux de lycéens (en particulier dans les classes terminales) à Orléans, associée à l'exploitation historienne de statistiques de longue durée (Prost 1986), nos pronostics dubitatifs sur une avancée continue de la démocratisation du recrutement de l'Ecole, qui, à l'époque de nos enquêtes artisanales des années 60, n'avaient pu revêtir chez nous que la forme de simples conjectures sociologiques.

\section{Notes}

${ }^{1}$ Claude Lévi-Strauss le disait dans l'épigraphe nostalgique qu'il plaçait en tête du premier volume de son Anthropologie structurale (1958), abandon qui s'expliquait, ajoute-t-ill, "moins par l'ingratitude que par la triste persuasion où nous sommes, que l'entreprise excéderait aujourd'hui nos forces".

${ }^{2}$ Comme le rappelait plaisamment Alain Desrosières (1982:168-166), commentant cette technique de I'INSEE, le "redressement " pratiqué à la seule fin de modéliser un système de variables conduit à examiner les corrélations entres variables sur " une population fictive où la Lozère comprend en partie une grande métropole urbaine et où Paris est peuplé d'une fraction appréciable d'agriculteurs ou de salariés agricoles...". Sur ce point, cf. Passeron 1991:126-133.

${ }^{3}$ Voir en particulier dans le débat qui est suivi d'un textbook les textes de Georges Navel, Jean Guehenno ou Sartre.

4" Conservatisme et novation à l'Université " (Antoine \& Passeron 1966) où, sans m'attarder à l'antique justification de la valeur charismatique de la relation entre "maître " et "disciples " que tentaient encore de maintenir les philosophes traditionalistes (par exemple, Gusdorf 1963), j'opposai à l'analyse monolithique de Vermot-Gauchy les hypothèses et les calculs réalistes du Great Britain Committee on Higher Education nommé par le Premier Ministre britannique sous la présidence du Lord Robbins (1963).

5Je l'ai développé ensuite à l'Université d'Alger dans un séminaire qui a donné lieu à un document ronéoté retranscrit à la même époque (1968) par Jean-Pierre Briand alors en poste à la Faculté et à l'Institut d'Etudes Politiques: "Le chiffre et le sens "; je ne l'ai retrouvé que récemment, avec ses tableaux, graphiques, courbes et modèles simulant un processus de mesures successives dans le temps, exemplifiées par des données d'enquêtes sur la "sélection scolaire ", afin d'analyser plusieurs hypothèses susceptibles d'expliquer la distribution, entre classes ou catégories sociales, des degrés de performance au long d'un processus d'élimination différentielle, en supposant au départ du processus des aptitudes inégales entre les individus; mais on ne peut pas supposer une inégalité entre catégories statistiques dès lors qu'elles contiennent chacune une population assez nombreuse pour qu'on soit certain d'y retrouver, à la naissance, la même distribution gaussienne, entre les individus de chaque catégorie, des aptitudes à acquérir des aptitudes.

${ }^{6}$ La synthèse des travaux statistiques, ethnologiques et sociologiques réalisé par l'ARDES a été publiée en commun par leurs responsables sous le titre Travail et travailleurs en Algérie, par P. Bourdieu, A. Darbel, J.-P. Rivet et C. Seibel (1963). L'enquête sociologique sur les "centres de regroupement " menée 
dans ce même cadre en 1961 ne pourra être publiée que plus tard par Pierre Bourdieu et Abdelmalek. Sayad (1964).

${ }^{7}$ Cf. par exemple Thélot (1982).

8 J.-P. Benzécri et collab. 1973. La systématisation clairement " réaliste " des objets de connaissance par Benzécri, mathématicien, dont je connaissais le "thomisme" de conviction depuis nos discussions sur Spinoza à l'ENS dans les années 50 , est aussi fort utile en ce qu'elle oblige à une utilisation circonspecte de cet instrument, dont à la même époque, tant de sociologues s'entichaient aveuglément, souvent jusqu'au contre-sens d'interprétation, laissé à la charge de l'étudiant ou du lecteur, sur la part d'inertie qu'extrayait chacune des modalités de l'analyse sur chacun des axes créés par le calcul vectoriel. Cf. par réaction à ces abus et à leurs conséquences pédagogiques dans la formation des chercheurs, la

présentation critique du sens des plans factoriels qui étaient utilisés dans les enquêtes du GIDES en 197778 sur l'introduction de l'audio-visuel dans les bibliothèques publiques : résultats synthétisés dans L'œil à la page (Grumbach, Passeron et al. 1979).

${ }^{9}$ Utilisé par Bourdieu \& Passeron (1964).

${ }^{10} \mathrm{C}$ 'est ce calcul probabiliste des chances qui a permis de suivre, à l'occasion des recensements opérés ultérieurement par l'INSEE, une démocratisation mitigée et vite ralentie du recrutement scolaire, sinon aux différents degrés en tout cas dans les filières les plus prometteuses de réussite professionnelle - Grignon \& Passeron 1970; Bourdieu \& Passeron 1972 ; Passeron 1986a. La « démocratisation » ne s'est guère manifestée que par une " translation vers le haut » de la structure globale de l'inégalité des chances objectives d'accès. Étant donné que, dans cette translation, les groupes les plus favorisés approchaient, dans les années 80, de la certitude de voir leurs enfants scolarisés dans l'enseignement supérieur, le léger rétrécissement de l'éventail des chances d'entrée à l'Université n'encourageait guère les " espérances subjectives " des enfants issus des catégories défavorisées d'y accéder un jour, et donc l'aspiration à une scolarisation qui continuait, dans ces groupes, à être vécue subjectivement comme «improbable » et inutile pour eux; mais il a abouti, comme on l'a vu ces vingt dernières années, à l'échec pédagogique des ambitions démocratiques de l'Ecole de masse et à la crise corrélative des métiers d'enseignement. Cf. F. Dubet et son analyse du déclin du «programme institutionnel » de démocratisation et d'intégration (2002).

${ }^{11}$ Cette relativisation du sens d'une « composition sociale " n'est exigible, bien sûr, que lorsque la disparité entre les volumes des populations de référence ou d'origine est forte : on retrouverait au contraire dans les simples pourcentages du sex-ratio de différentes catégories le même ordre de grandeur que par le calcul de la « probabilité objective » de chacun des deux sexes d'être représenté dans ces mêmes catégories. On rapporte, en effet, par les deux méthodes de calcul, les mêmes effectifs à une même base : à savoir les $50 \%$ de garçons ou de filles à la naissance. Mais, on le voit aussitôt, la différence réapparaît si on veut calculer les " probabilités conditionnelles ", pour les hommes et les femmes, d'être représentés dans les sous-catégories d'une catégorie, une fois supposée acquise leur entrée dans cette catégorie d'ensemble: par exemple lorsqu'on calcule en termes de « probabilités conditionnelles » les chances de survie, chez les hommes et les femmes, après un âge donné ; ou les chances de survie scolaire après une longueur d'études donnée ; de même pour les probabilités de "mortalité scolaire " différentielle dans un cursus ou dans une filière déterminée ; ou encore dans les réitérations successives d'une pratique culturelle, après que celle-ci ait été essayée une première fois. Tout processus temporel doit être questionné, au moyen d'instruments statistiques adéquats, sur l'essorage différentiel des catégories qu'il sélectionne progressivement, sous peine de voir biaisée la mesure des relations entre les critères de la catégorisation, lorsqu'on se contente de mesurer synchroniquement ces relations, en oubliant la sous-sélection et la sur-sélection continue de catégories définies par des critères qui jouent un rôle actif dans l'élimination d'une partie de la population.

\begin{abstract}
${ }^{12}$ Nous découvrîmes avec étonnement cet auteur - dans l'éloignement de la culture ouvrière anglaise comme dans sa proximité sociologique aux attitudes des cultures populaires en France - pendant que nous préparions Les héritiers, dans l'édition en livre de poche de l'essai, paru l'année précédente chez Chatto \& Windus, The Uses of Literacy (1958) dont j'introduisis plus tard la traduction française aux Editions de Minuit (1970).
\end{abstract}

${ }^{13}$ Le premier article de Bernstein que nous lûmes figurait dans le British Journal of Sociology. (1958) Plus tard, dans les colloques où nous le rencontrâmes et à Paris où il fut plusieurs fois invité à l'EHESS, nous pûmes confronter longuement nos analyses respectives du rôle de l'apprentissage du langage dans les divers milieux familiaux en discutant son ouvrage Class, Codes and Control (1971).

${ }^{14}$ Bourdieu, Passeron, de Saint-Martin (1965).

${ }^{15}$ Bourdieu, Chamboredon et Passeron, 1968 (2ème édition abrégée, 1970).

${ }^{16}$ Cette évolution a conduit l'un des auteurs à des rectifications et des précisions sur la validité de la thèse présentée dans La reproduction (par exemple dans Passeron 1986, puis à la présentation systématisée des schèmes les plus caractéristiques de l'argumentation dans les sciences sociales considérées comme sciences historiques (Passeron 1991) ainsi que dans les articles de la décennie qui suit, tandis que Pierre Bourdieu prolongeait, approfondissait et radicalisait la théorie de la domination symbolique esquissée dans les ouvrages sur l'éducation écrits dans les années 60, et cela dès La distinction : critique sociale du jugement (1979), et Questions de sociologie (1980) en passant par Homo academicus (1984), Noblesse d'état (1989), Les règles de l'art (1992) jusqu'aux Méditations pascaliennes (1997) qui énonçaient clairement les rapports entre théorie sociologique et philosophie pour placer la première au principe de la 
seconde dont elle englobait la vérité anthropōlogique.

${ }^{17}$ UNEF 1966.

${ }^{18}$ Les résultats des premières enquêtes réalisées de 1953 à 1955 et commentées par A. Sauvy et A. Girard dans Population seront suivis dans les années 60 d'enquêtes de suivis de cohortes mieux outillées et plus représentatives publiées au fur et à mesure dans la même revue sous la direction d'Alain Girard et sous les co-signatures de P. Clerc, H. Bastide et G. Pourcher (1970).

${ }^{19}$ Puisque je les ai commentés plus en détail dans un article récent (2003).

20 Je pense en particulier à un article célèbre de l'économiste Maurice Allais (1953). Sa critique de l'application mécaniquement mathématique de la courbe de Bernoulli, qui suppose l'ajout de "l'axiome d'indépendance " de Savage et de "l'axiome de substitution " de Samuelson, s'appuie sur le recours à des faits ou des expériences psychologiques et sociologiques qui le conduisent à distinguer clairement, pour les besoins explicatifs d'une science empirique, les espérances subjectives des acteurs déformant les probabilités objectives ou les ignorant. II faut donc se donner les moyens de connaître les espérances subjectives pour rendre compte de leurs comportements réels : "Seul un statisticien de profession peut se faire une idée correcte de ce que signifie une probabilité égale à une chance sur cent. Il y a même des cas où la notion de probabilité objective disparaît complètement sans qu'il en soit de même de celle de probabilité subjective. Ces cas correspondent aux coups isolés. " (p 508).

${ }^{21}$ C'est ce que j'ai tenté de faire dans la conception d'une enquête appliquée sur les étudiants en médecine, commandée par la Faculté pour ses besoins docimologiques et réalisée en collaboration avec M. de Saint Martin (1964) En introduisant dans le questionnaire un tableau permettant aux sujets de cocher d'un oui ou d'un non les membres de leur famille étendue qui, à leur connaissance, avaient fait des études supérieures (père, mère, grands parents et arrières grands parents ; sœurs, frères et cousins, oncles et tantes) on pouvait identifier l'espace social où s'étaient formées leurs espérances subjectives de fréquenter l'école et d'y réussir avec les aspirations qui en découlaient. Le dépouillement révélait, par exemple, que les enfants d'origine populaire ou petite-bourgeoise, rarissimes dans ces études, mais pourtant présents à la Faculté de médecine comme étudiants atypiques, avaient eu dans leur famille étendue le plus haut score familial sur cet indice, trois fois supérieur à celui de la valeur moyenne de la scolarisation probable dans la même catégorie socio-professionnelle lorsqu'on la considère dans son ensemble.

${ }^{22} \mathrm{Cf}$. sur la question méthodologique du " bon usage " de la comparaison en sociologie comme dans toutes les sciences historique v. Passeron 1982 ; ou, plus récemment, Passeron 2000 : 13-33.

\section{References}

ALLAIS M., 1953, "L'homme rationnel devant le risque : critique des postulats et axiomes de l'Ecole américaine", Econometrica, 21 (4) Paris.

ANTOINE G. \& Passeron J.-C., 1966, "Conservatisme et novation à l'Université " in La réforme de l'Université, Paris, Calmann-Lévy.

BEAUDELAIRE C., 1887, Mon cœur mis à nu, (LXXVII), Paris, Maison Quantin.

BENZÉCRI J.-P. et collab., 1973., L'analyse des données ( $2^{\mathrm{e}}$ vol), Paris, Dunod.

BOURDIEU P. \& Passeron J.-C., 1964, Les Héritiers, Paris, Minuit.

BOURDIEU P. \& Passeron J.-C., 1972, La reproduction, Paris, Minuit.

BOURDIEU P. \& Sayad A., 1964, Le déracinement, Paris, Minuit.

BOURDIEU P., 1979, La distinction : critique sociale du jugement, Paris, Minuit.

BOURDIEU P., 1980, Questions de sociologie, Paris, Minuit.

BOURDIEU P., 1984, Homo academicus, Paris, Minuit.

BOURDIEU P., 1989, Noblesse d'état, Paris, Minuit.

BOURDIEU P., 1992, Les règles de l'art : genèse et structure du champ littéraire, Paris, Seuil.

BOURDIEU P., 1997, Méditations pascaliennes Paris, Seuil.

BOURDIEU P., Chamboredon J.-C. et Passeron J.-C., 1968 (2 ${ }^{\text {ème }}$ édition abrégée, 1970), Le métier de sociologue : préalables épistémologiques, Paris, Mouton-Bordas.

BOURDIEU P., Darbel A., Rivet J.-P., Seibel C., 1963, Travail et travailleurs en Algérie, Editions Mouton, La Haye. 
BOURDIEU P., Passeron J.-C. et de Saint-Martin M., 1965, Rapport pédagogique et communication, ParisLa Haye, Mouton.

CHAPOULIE J.-M., 1991, " La seconde fondation de la sociologie française, les Etats-Unis et la classe ouvrière ", Revue française de sociologie, 32 (3), p. 321-364.

CLERC P., Bastide H. et Pourcher G., 1970, 'Population' et l'enseignement, Paris, PUF-INED.

DESABIE J., 1966, Théorie et pratique des sondages, Paris, Dunod.

DESROSIÈRES A., 1982, "Réflexions sur la portée sociologique des diverses phases du travail statistique ", Actes de la Journée Sociologie et Statistique, Paris, INSEE/SFS.

DUBET F., 2002, Le déclin de l'institution, Paris, Le Seuil.

DURKHEIM E., 1895, Règles de la méthode sociologique, Paris, F. Alcan.

GREAT BRITAIN COMMITTEE ON HIGHER EDUCATION (appointed by the Prime Minister under the chairmanship of Lord Robbins), 1963, Londres, Her Majesty's Stationery Office.

GRIGNON C. \& Passeron J.-C., 1970, Expériences françaises avant 68, Paris, OCDE.

GRIGNON C. \& Passeron J.-C., 1989, Le savant et le populaire : misérabilisme et populisme en sociologie et en littérature, Paris, Le Seuil.

GRUMBACH M., Passeron J.-C. et al., 1979, L'œil à la page, Paris, Ed. BPI.

GUSDORF G., 1963, Pourquoi des professeurs ?, Paris, Payot.

HALBWACHS M., 1935, "La statistique en sociologie " in M. Halbwachs : Classes sociales et Morphologie, Paris, Éditions de Minuit, 1972, p. 329-348.

LÉVI-STRAUSS C., 1958, Anthropologie structurale, Paris, Plon.

PASSERON J.-C., 1982, “' L'inflation des diplômes : remarques sur l'usage de quelques concepts analogiques en sociologie ", Revue française de sociologie (23).

PASSERON J.-C., 1986, “ Hegel ou le passager clandestin : la reproduction sociale et l'histoire ", Esprit, $\mathrm{n}^{\circ}$ 115, p. 63-81.

PASSERON J.-C., 1986a, Histoire des Universités en France, Toulouse, Privat.

PASSERON J.-C., 1991, Le raisonnement sociologique : un espace non-poppérien du raisonnement naturel, Paris, Nathan.

PASSERON J.-C. (dir.), 1999, Richard Hoggart en France, Paris, Ed. de la BPI.

PASSERON J.-C., 2000, "Analogie, connaissance et poésie ", Revue européenne des sciences sociales, $\mathrm{n}^{\circ} 117$.

PASSERON J.-C., 2002, “Mort d'un ami, disparition d'un penseur ", Revue européenne des sciences sociales, Genève, Droz, $n^{\circ} 125$, décembre, p. 77-125.

PASSERON J.-C., 2003, "Consommation et réception de la culture: la démocratisation des publics, in Donnat O. \& Tolila P. (dir.), Le(s) public(s) de la culture, Paris, Presses de Sciences Po, pp. 359-390.

PROST A., 1986, L'enseignement s'est-il démocratisé ? Paris, PUF.

ROBERT J.-L., 2002, “Quand les sciences sociales entrent dans I'histoire ”, Journée du 22 mai 2002.

THÉLOT C., 1982, Tel père, tel fils : position sociale et origine familiale, Paris, Dunod.

UNEF / Association Fédérative Générale des Étudiants de Strasbourg, 1966, "De la misère en milieu étudiant, considérée sous ses aspects économique, politique, psy(s)chologique, sexuel et notamment intellectuel, et de quelques moyens pour y remédier ", Étudiants de France (Supplément spécial au n¹6), Strasbourg.

VERMOT-GAUCHY M., 1965, L'Education Nationale dans la France de demain : Futuribles, Ed. du Rocher, Monaco. 